\title{
The Impact of Adjuvant Chemotherapy on Health-Related Quality of Life (HR-QoL), A Prospective Multicenter Measurement Study
}

ISSN: 2637-7632

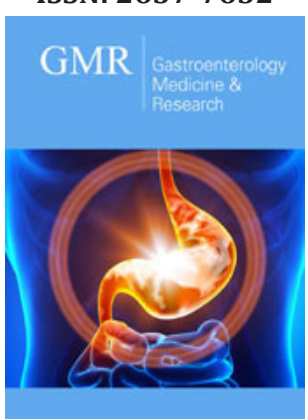

*Corresponding author: Antoine Abi Abboud, Professor of Gastroenterology, Department of Gastroenterology, Faculty of Medical Sciences, Lebanon

Submission: 啙 October 14, 2019

Published: 㘹 November 07, 2019

Volume 3 - Issue 5

How to cite this article: Naim S Y, Ali $\mathrm{H}$ A, Antoine Abi $\mathrm{A}^{*}$ and Evelyne G H. The Impact of Adjuvant Chemotherapy on Health-Related Quality of Life (HR-QoL), A Prospective Multicenter Measurement Study. Gastro Med Res. 3(5). GMR.000575. 2019.

DOI: 10.31031/GMR.2019.03.000575

Copyright@ Antoine Abi Abboud, This article is distributed under the terms of the Creative Commons Attribution 4.0 International License, which permits unrestricted use and redistribution provided that the original author and source are credited.

\begin{abstract}
Naim S Yarak ${ }^{1}$, Ali H Alayane ${ }^{2}$, Antoine Abi Abboud ${ }^{3 *}$ and Evelyne G Helou ${ }^{4}$
${ }^{1}$ Department of Urologic Surgery, Lebanese University, Faculty of Medical Sciences, Lebanon

${ }^{2}$ Department of Orthopedic Surgery, Lebanese University, Faculty of Medical Sciences, Lebanon

${ }^{3}$ Department of Gastroenterology, Lebanese University, Faculty of Medical Sciences, Lebanon

${ }^{4}$ Department of Hematology-Oncology, Lebanese University, Faculty of Medical Sciences, Lebanon
\end{abstract}

\begin{abstract}
Introduction: Chemotherapy is one of the most commonly employed cancer treatments since its use provides a strong evidence for disease-free survival as well as overall survival for several cancer types. This type of treatment is associated with detrimental side effects that are difficult to tolerate and that impact the quality of life (QOL) of patients on different levels. As more patients undergo adjuvant chemotherapy, there has been a growing interest in investigating its impact on health-related quality of life (HRQOL). To our knowledge, most published literature focuses on HRQOL at specific time points during chemotherapy and only limited data describes the dynamics of HRQOL starting before treatment, during the complete duration and after completion of chemotherapy.
\end{abstract}

Objective: The objective of this study is to evaluate the impact of adjuvant chemotherapy on a patient's quality of life before the start, half-way through, at the end and 3 months after the end of chemotherapy and to examine whether individual differences in quality of life among adjuvant chemotherapy patients could be accounted for by selected demographic and medical characteristics.

Methods: Seventy-eight patients diagnosed with solid tumors were interviewed before starting their first chemotherapy at Sacré-Coeur hospital, Rafic Hariri University Hospital, Saint Joseph Hospital, Family Medical Center between January 1st, 2016 and December 31st, 2018. Data collection included the EORTC core quality of life questionnaire (QLQ_C30) which was filled before, during, at the end and 3 months after undergoing adjuvant chemotherapy. The statistical analysis was done using the SPSS program and the strength and the direction of association that exists between variables were measured using Pearson correlation. Repeated measure ANOVA was used to compare results of the QLQ-C30 scores test in different categories of patients.

Result: The QLQ-C30 scores showed statistically significant changes over time, $F(1.889,145.462)=85.632$, $\mathrm{p}<0.001$, partial $\eta 2=0.527$, with $\mathrm{QLQ}-\mathrm{C} 30$ score increasing from $46.37 \pm 10.36$ pre-intervention to $66.56 \pm 15.70$ during adjuvant chemotherapy, then decreasing to $64.21 \pm 15.49$ directly post-intervention, and further decreasing to $53.29 \pm 12.563$ months after the end of the treatment. There was no statistically significant correlation between age at diagnosis, comorbidities, number of chemotherapy cycles, type of cancer and QLQ-C30 scores before, during and after adjuvant chemotherapy.

Conclusion: The impact of adjuvant chemotherapy on HR-QoL was transient and less substantial than the process of adaptation to the disease. Moreover, after completion of cytotoxic treatment, there was no lasting adverse effect on QoL. These results can guide patients on treatment options and informed decision-making can be made by the clinician.

Keywords: Cancer; HR-QoL; Adjuvant chemotherapy

Abbreviations: HR-QoL: Heath Related Quality of Life; QoL: Quality of Life

Introduction

Patients face the diagnosis of cancer as one of the most traumatic life experiences. In fact, regardless of prognosis, the occurrence of cancer results in major changes in a patient's life perspective and expectations, including his professional and social role. The use of current therapies including surgery, radiation therapy, chemotherapy, biological and immune therapy, results in the cure of more than $70 \%$ of patients diagnosed with cancer. Chemotherapy is one of the most commonly employed cancer treatments since its use provides a strong 
evidence for disease-free survival as well as overall survival for several cancer types [1-4]. This type of treatment is associated with detrimental side effects that are difficult to tolerate and that impact the quality of life (QOL) of patients. In addition, as more patients undergo adjuvant chemotherapy, there has been a growing interest in investigating its impact on health-related quality of life (HRQOL).

QOL is considered a robust predictor of treatment success and survival, and consequently it is associated with utmost prognostic value. QOL can guide healthcare decisions through delineating the side effects of treatments and should be communicated with patients to help them understand the course of their disease. The constituents of health-related quality of life (HRQOL) are based on the World Health Organization (WHO) definition of health as a "status of complete physical, mental and social well-being and not merely the absence of illness" and include physical functioning, mental status, and social elements [5,6]. In addition, HRQOL is related to individual factors such as personality and coping mechanisms and external aspects including social relationships, surrounding environment, and life challenges [7]. According to the world health organization (WHO), the quality of life is defined as the "individual's perception of their position in life in the context of the culture and value systems in which they live and in relation to their goals, expectations, standards and concerns" The most frequently reported side effects during and after chemotherapy cycles include nausea, vomiting, loss of appetite, hair loss and fatigue [8,9]. In addition to the physical capacity, patients report a weakening in cognitive function related to memory, processing speed and executive function [4]. These associated toxicities and cognitive deficits are of utmost concern since they can impact negatively the patient's psychological and emotional stability and consequently impact his readiness to endure efficaciously the treatment $[9,10]$. Moreover, chemotherapy treatment can be administered for an extended duration and requires recurrent hospitalization which intensifies the burden on cancer patients [11]. Along these lines, chemotherapy results in physical, psychological, emotional, economic, and social alterations among cancer patients, affecting their overall QOL [12]. To our knowledge, most published literature focuses on HRQOL at specific time points during chemotherapy and only limited data describes the dynamics of HRQOL starting before treatment, during the complete duration and after completion of chemotherapy.

Consequently, our overall Aim is to evaluate the impact of adjuvant chemotherapy on a patient's QOL at different time points:
A. Before the start of chemotherapy
B. Half-way through chemotherapy
C. At the end of chemotherapy
D. 3 months after the end of chemotherapy

\section{Methods}

\section{Patients}

We enrolled 78 patients diagnosed with solid malignancies who were planned to receive their first adjuvant chemotherapy session in three secondary care centers between January 1 1', 2016 and December $31^{\text {st }}$, 2018. Patients who satisfied the inclusion criteria signed an informed consent.

\section{Categorization}

The aim of the study was explained to each patient. The latter was informed that he is expected to answer the questionnaire 4 times: At the beginning, during, at the end of the chemotherapy cycles and 3 months after completion of the cycles. The first three questionnaires were filled out by face to face interviews. The patient was informed that he/she will be asked to answer the last questionnaire after 3 months from the end of the chemotherapy by phone.

\section{EORTC core quality of life questionnaire (QLQ_C30): should I include an overview?}

\section{Statistics}

The statistical analysis was done using the SPSS program and the strength and the direction of association that exists between variables were measured using Pearson correlation. Repeated measure ANOVA was used to compare results of the QLQ-C30 scores test in different categories of patients. The level of $<0.05$ was considered as significant.

\section{Result}

\section{Patients characteristics}

For a total of 78 patients: The median age at diagnosis was 52(range 29-77). Most of the recruited patient, 54(69.2\%), had breast cancer, while $9(11 \%)$ had colon cancer, and 15(19.8\%) were distributed among all of the other cancer types such as lung cancer, pancreatic cancer, etc...To treat the latter cancers 16 different adjuvant chemotherapy were used in the studied sample. These regimes varied from using one chemotherapy, such as using cisplatin to treat cervical and neuroendocrine tumors, to using combination or sequential therapy, such as using Doxorubicin and Cyclophosphamide, Docetaxel to treat poorly differentiated breast cancer. The median number of cycles used to treat cancer is 8 cycles (1-20). Patients demographics showed that only $11(14.1 \%)$ of the patients had previous surgeries unrelated to cancer, $10(12.8 \%)$ smokes, and 4(5.1\%) consumes alcohol. Additionally, comorbidities such as hypertension (HTN), diabetes mellitus (DM) were reported to be $29.5 \%$ and $15.4 \%$ respectively.

\section{Impact of adjuvant chemotherapy on health-related quality of life}

The QLQ-C30 scores showed statistically significant changes over time, $F(1.889,145.462)=85.632, p<0.001$, partial $\eta 2=0.527$, with QLQ-C30 score increasing from $46.37 \pm 10.36$ pre-intervention to $66.56 \pm 15.70$ during adjuvant chemotherapy, then decreasing to $64.21 \pm 15.49$ directly post-intervention, and further decreasing to $53.29 \pm 12.563$ months after the end of the treatment. Results are shown in Figure 1. There was no statistically significant correlation between age at diagnosis, comorbidities, number of chemotherapy cycles, type of cancer and QLQ-C30 scores before, during at the end and 3 months after adjuvant chemotherapy as shown in Table 1. 


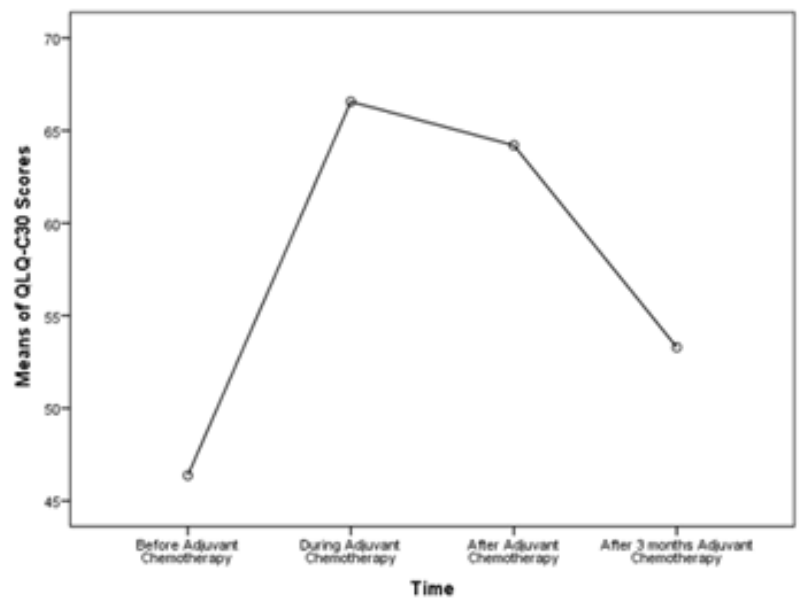

Figure 1: Mean plot of the QLQ-C30 scores before, during, directly after, and after 3 months of receiving adjuvant chemotherapy among all cancer patients.

Table 1: $\mathrm{p}$ value of the QLQ-C30 scores before, during, directly after, and after 3 months of receiving adjuvant chemotherapy among all the study's variables.

\begin{tabular}{|c|c|c|c|c|}
\hline & Age at Diagnosis & $\begin{array}{c}\text { Comorbidities } \\
\text { HTN }\end{array}$ & Comorbidities DM & $\begin{array}{c}\text { Number of Chemotherapy } \\
\text { Cycles }\end{array}$ \\
\hline QLQ-C30 Score Before Adjuvant Chemotherapy & 0.733 & 0.876 & 0.17 & 0.762 \\
\hline QLQ-C30 Score during Adjuvant Chemotherapy & 0.591 & 0.424 & 0.323 & 0.824 \\
\hline QLQ-C30 Score directly after Adjuvant Chemotherapy & 0.94 & 0.26 & 0.264 & 0.651 \\
\hline QLQ-C30 Score 3 months Adjuvant Chemotherapy & 0.221 & 0.787 & 0.066 & 0.409 \\
\hline
\end{tabular}

\section{Discussion}

Our study showed that the QLQ-C30 scores resulted in statistically significant changes over time $(\mathrm{p}<0.001)$ with QLQ-C30 score increasing from $46.37 \pm 10.36$ pre-intervention to $66.56 \pm 15.70$ during adjuvant chemotherapy, then decreasing to $64.21 \pm 15.49$ directly post-intervention, and further decreasing to $53.29 \pm 12.563$ months after the end of the treatment. Thus, patients' assessment of their QL is influenced by adaptation to the disease and its treatment over time. To our knowledge, the majority of researches done on this topic have concentrated on the active treatment period. For instance; Cyclophosphamide, methotrexate and 5-fluorouracil (CMF) chemotherapy and tamoxifen was compared with tamoxifen alone in three studies, and one study compared differing durations of CMF. In general, these studies demonstrated that chemotherapy temporarily impaired overall HRQL, physical and emotional domains, and specific symptoms, but the effects were transient and improved rapidly after chemotherapy ended [13]. However, some others focused only on the QoL post adjuvant therapy inasmuch as much less is known about quality of life following completion of adjuvant chemotherapy. To note that although a number of studies have investigated quality of life among breast cancer survivors, relatively few have focused specifically on women treated previously with adjuvant chemotherapy. In one of the first studies to address this issue, Knob surveyed 28 women with breast cancer who had completed a variety of adjuvant chemotherapy regimens an average of 28 months previously (range 2-60 months) and 50 women still receiving adjuvant chemotherapy. Analyses indicated that current chemotherapy patients generally reported greater distress about specific symptoms (e.g., nausea and fatigue) than former chemotherapy patients. In contrast, former chemotherapy patients score higher on two indices of psychological distress (concerns about breast loss and concerns about survival) than current chemotherapy patients [7]. By analyzing the collected data and the results of our study, we concluded that QLQ-C30 scores were statistically non-significant between the cancer types and different treatment used before, during and at the end of chemotherapy.

This contrasts with expert review results done by Grimison and Stockler regarding the impact of adjuvant chemotherapy on quality of life in early stage breast cancer patients. Chemotherapy regimens including an anthracycline were compared with cyclophosphamide, methotrexate and 5-fluorouracil (CMF) in four different randomized controlled trials. Anthracycline-containing regimens resulted in worse overall well-being, nausea, hair loss and functional impairment than CMF during treatment, but these effects were transient and recovered rapidly after treatment ended. Even though that it was found by the Early Breast Cancer Trialists' Collaborative Group overviews that anthracycline-containing regimens give better disease-free and overall survival than CMF. We concluded that there was no statistically significant correlation between HTN and DM and QLQ-C30 scores before, during and after adjuvant chemotherapy which was in line with other studies done on this topic. Just to mention a few, a prospective study was done by Chean et al. where EORTC QLQ-C30 questionnaire was given to 32 
female patients with breast cancer to fill in prior to (baseline) and after the third cycle of chemotherapy.

They reported a significant lower global health status $(\mathrm{P}<0.01)$ and significant higher symptoms of nausea and vomiting $(\mathrm{P}<0.01)$, loss of appetite $(\mathrm{P}=0.028)$ and diarrhea $(\mathrm{P}=0.026)$ after the third cycle of chemotherapy as compared to baseline. They most importantly noted that comorbidities had no clear association with global health status in these patients ( $\mathrm{P}>0.05)$. Also Chean et al. showed that the influence of a given comorbid condition will vary depending on where a patient is located within the cancer care trajectory. According to a study done by Wolf (2005), patients with comorbidity such as diabetes mellitus did not appear to worsen breast-cancer specific outcome which was in line with this study result. However, Mao (2007) observed that symptom burden among cancer survivors in their study is substantial and impacted by other comorbidities. This study found that there was no correlation between age and quality of life. Watters (2003) encouraged this finding and reported that quality of life of young ( $<65$ years old) and older ( $\geq 65$ years old) patients had similar decline as in global health status. Similar results also have been reported by Mols (2005) which concluded that age is not a predictor of quality of life. Moreover, we found no statistically significant correlation between number of chemotherapy cycles and QLQ-C30 scores at all reported time points. This is in contrary to a study done by Hürny et al. discussing the impact of adjuvant therapy on quality of life in women with node-positive operable breast cancer. Patients who received more doses of chemotherapy reported slower improvement in QL after initial diagnosis and surgery. This finding shows that it is important to assess both QL and efficacy when making treatment decisions [14].

\section{Conclusion}

The results of this research generally indicate that cancer patients experience problems in multiple quality of life domains while undergoing adjuvant chemotherapy. This impact was transient and less substantial than the process of adaptation to the disease. Moreover, after completion of cytotoxic treatment, there was no lasting adverse effect on QoL. These results can guide patients on treatment options and informed decision-making can be made by the clinician. Although QoL is a subjective perception of cancer patients' symptoms, function and side effects of the treatment, it may help clinicians in making treatment decisions and determine the best ways to support and improve the quality of life of cancer patients during the difficult times of their disease and on-going cancer treatments. From what preceded, we conclude that more efforts should be directed toward instigating methods to promote treatment facilities, assist recuperation and palliative care to support cancer patient to cope for a better recovery, improve their quality of life and help them reintegrate in their social sphere.

\section{References}

1. Broeckel JA, Jacobsen PB, Balducci L, Horton J, Lyman GH (2000) Quality of life after adjuvant chemotherapy for breast cancer. Breast Cancer Res Treat 62(2): 141-150.

2. Lim S, Park SH, Park HK, Hur MH, Oh SJ, et al. (2015) Prognostic role of adjuvant chemotherapy in Node-Negative (N0), Triple-Negative (TN), Medullary Breast Cancer (MBC) in the Korean population. PloS one 10(11): e0140208.

3. Winter-Roach BA, Kitchener HC, Lawrie TA (2012) Adjuvant (postsurgery) chemotherapy for early stage epithelial ovarian cancer. Cochrane Database Syst Rev 3: CD004706.

4. Dwek MR, Rixon L, Simon A, Hurt C, Newman S (2015) Examining the effects of adjuvant chemotherapy on cognition and the impact of any cognitive impairment on quality of life in colorectal cancer patients: Study protocol. BMC Psychol 3: 43.

5. Berzon R, Hays RD, Shumaker SA (1993) International use, application and performance of health-related quality of life instruments. Qual Life Res 2(6): 367-368.

6. Fitzpatrick R, Davey C, Buxton MJ, Jones DR (1998) Evaluating patientbased outcome measures for use in clinical trials. Health Technol Assess 2(14): 1-74.

7. Martinez-Martin P (2017) What is quality of life and how do we measure it? relevance to Parkinson's disease and movement disorders. Mov Disord 32(3): 382-392.

8. Coates A, Abraham S, Kaye SB, Sowerbutts T, Frewin C, et al. (1983) On the receiving end-patient perception of the side-effects of cancer chemotherapy. Eur J Cancer Clin Oncol 9(2): 203-208.

9. Carelle N, Piotto E, Bellanger A, Germanaud J, Thuillier A, et al. (2002) Changing patient perceptions of the side effects of cancer chemotherapy. Cancer 95(1): 155-163.

10. Wefel JS, Vardy J, Ahles T, Schagen SB (2011) International cognition and cancer task force recommendations to harmonize studies of cognitive function in patients with cancer. Lancet Oncol 12(7): 703-708.

11. Rashid YA, Ghafoor ZA, Masood N, Mehmood T, Awan S, et al. (2012) Psychosocial impact of cancer on adult patients. J Pak Med Assoc 62 (9): 905-909.

12. Akin S, Can G, Aydiner A, Ozdilli K, Durna Z (2010) Quality of life, symptom experience and distress of lung cancer patients undergoing chemotherapy. Eur J Oncol Nurs 14(5): 400-409.

13. Grimison PS, Stockler MR (2007) Quality of life and adjuvant systemic therapy for early-stage breast cancer. Expert Rev Anticancer Ther 7(8): 1123-1134.

14. Hürny C, Bernhard J, Castiglione-Gertsch M, Coates AS, Peterson HF, et al. (1996) Impact of adjuvant therapy on quality of life in women with node-positive operable breast cancer. Lancet 347(9011): 1279-1284. 